\title{
RDW-CV E RDW-SD EM GATOS DOMÉSTICOS SAUDÁVEIS
}

\author{
(RDW-CV And RDW -SD in healthy domestic cat) \\ Bruna Brito Oliveira, Simone Sorgatto, Kelly Cristina da Silva Godoy, Gustavo Gomes de Oliveira, Alda \\ Izabel de Souza \\ ${ }^{1}$ Correspondência: bruoliveira_vet@hotmail.com
}

RESUMO: O objetivo do presente estudo foi estabelecer intervalo de referência para valores de RDW-CV e RDW-SD em felinos clinicamente sadios. Amostras de sangue de 187 felinos jovens e adultos, clinicamente sadios e encaminhados para procedimentos cirúrgicos eletivos foram utilizadas. As amostras foram obtidas a partir da punção de veia jugular, armazenadas em tubos com EDTA e posteriormente processadas. A determinação do RDW foi realizada utilizando o analisador hematológico veterinário modelo pocH-100iV DIFF (Sysmex®). O intervalo de normalidade estabelecido para o RDW-CV foi de $16,8 \pm 1,84 \%$ e do RDW-SD $31,8 \pm 2,83$ fL. A análise estatística não mostrou diferença significativa entre sexo e faixa etária (teste $Z, p>0,05$ ). Os valores médios para RDW-CV e RDW-SD apresentados podem ser utilizados como referência para felinos domésticos saudáveis, sem raça definida, independente do sexo ou idade.

Palavras-chave: felinos; hematologia; valores de referência

ABSTRACT: The aim of this study was to establish reference range for RDW-CV values and RDW- SD in clinically healthy cats. Blood samples from 187 young cats and adults, clinically healthy and referred for elective surgical procedures were used. Samples were obtained from the jugular vein puncture, stored in tubes with EDTA, and processed further. The determination was performed using the RDW hematology analyzer veterinary POCH-100iV DIFF model (Sysmex®). The range of normal values established for the RDW-CV was $16.8 \pm 1.84 \%$ and the RDW-SD $31.8 \pm 2.83 \mathrm{fL}$. Statistical analysis showed no significant difference between sex and age group ( $Z$ test, $p>0.05$ ). Mean values for RDW-CV and RDW-SD presented can be used as a reference for healthy domestic cats mongrel, regardless of sex or age.

Key Words: felines; hematology; reference values 


\section{INTRODUÇÃO}

A amplitude de distribuição do diâmetro dos eritrócitos (RDW) é um parâmetro hematológico interpretado, em associação ao índice hematimétrico de Volume Corpuscular Médio (VCM), como indicador de anisocitose em uma população de eritrócitos. O RDW é calculado, eletronicamente, pelo método de impedância a partir da média do VCM, com obtenção do coeficiente de variação (RDW- $\mathrm{CV}$ ) e do desvio padrão (RDW-SD).

Embora a variação de tamanho dos eritrócitos seja habitualmente avaliada a partir do VCM, este parâmetro fornece uma visão restrita sobre a anisocitose, uma vez que é necessário um elevado percentual de células com volume alterado para refletir um VCM anormal (Zvorc et al., 2010). Já os dados do RDW geram respostas mais fidedignas sobre a intensidade de modificação no tamanho destas células (Silva, 2013). Valores mais baixos indicam população eritróide homogênea, enquanto valores elevados atestam excesso de heterogeneidade volumétrica da população (Zvorc et al., 2010).

Em seres humanos variação nos valores do RDW tem sido citado como biomarcador associado a eventos sépticos (Slaughter et al., 2013), acidente vascular cerebral (Saliba et al., 2015), doença cardiovascular (Hunziker et al., 2012) e doença arterial coronariana (Lappé et al., 2011). Na veterinária, estudos têm enfatizado a importância do RDW para confirmação da boa atividade medular mesmo em animais sem anemia (Saliba et al., 2015).

Contudo para a apropriada interpretação deste parâmetro e certeza diagnóstica é importante a determinação de intervalos de referência espécieespecífico e baseado em metodologias padronizadas. Neste contexto, este trabalho teve como objetivo estabelecer valores de referência para RDW-CV e RDW-SD de gatos domésticos saudáveis e verificar a influência do sexo e idade sobre esses parâmetros.

\section{MATERIAL E MÉTODOS}

O trabalho foi aprovado pela Comissão de Ética no Uso de Animais (CEUA) sob o protocolo número $688 / 2015$ e seguiu as recomendações da Sociedade Americana de Patologia Clinica Veterinária - ASVCP (Friedrichs et al., 2012).

Amostras sanguíneas de 187 gatos domésticos, sem raça definida, encaminhados ao Programa de Castração de Cães e Gatos do Centro de Controle de Zoonoses de Campo Grande, MS foram utilizadas. A seleção dos animais baseou-se na avaliação clínica prévia de machos e fêmeas. Animais com histórico ou sinal de enfermidade e fêmeas prenhez foram excluídos do trabalho. Para avaliação da influência da idade e devido à dificuldade na definição exata deste dado epidemiológico, os animais foram estratificados em jovens (até um ano) e adultos (igual ou maior que um ano), de acordo com a declaração do proprietário.

As amostras foram obtidas por punção da veia jugular utilizando seringas plásticas de $5 \mathrm{~mL}$, agulhas calibre $25 \times 7 \mathrm{~mm}$ e acondicionadas em tubos contendo anticoagulante EDTAK2 a 10\% (ácido etileno diamino tetracético), respeitando exatamente a proporção sangue/anticoagulante. Amostras com sinais de hemólise foram descartadas. A determinação do RDW foi realizada utilizando 0 analisador hematológico veterinário modelo pocH100iV DIFF (Sysmex®) submetido diariamente ao controle de qualidade pelos métodos L-J e X, de acordo com as recomendações do fabricante. $\mathrm{O}$ critério de inclusão da amostra baseou- 
se na observação de histogramas de eritrócitos com distribuição gaussiana normal.

Para avaliação de normalidade os dados foram submetidos ao teste estatístico de Kolmogorov-Smirnov $(p>0,1 \%)$. Para comparação entre gêneros (machos e fêmeas) e faixas etárias (menor de 1 ano e maior ou igual a 1 ano), os dados foram analisados por meio do teste $Z$ considerando $5 \%$ de significância. As análises estatísticas foram efetuadas utilizando o programa computacional BioEstat 5.0.

\section{RESULTADOS E DISCUSSÃO}

A interpretação correta dos resultados laboratoriais implica no conhecimento de valores de referência específicos por espécie, além de variações relativas a condições geográficas, de manejo, de raça, de idade, de sexo e até mesmo do laboratório que realizou as análises (Gonzalez et al., 2001; Zvorc et al., 2010). Intervalos de referência para RDW calculados para indivíduos saudáveis diferem quando obtido por analisadores de fabricantes diferentes e, às vezes, até mesmo em modelos diferentes do mesmo fabricante (Caporal e Comar, 2013).

Os valores das médias, desvio padrão e valores mínimos e máximos de RDW-CV e RDW-SD estão apresentados na tabela 1 . O teste de Kolmogorov-Smirnov verificou 0 pressuposto de normalidade dos dados e constatou que as variáveis apresentaram distribuição paramétrica, indicando a homogeneidade das amostras.

Segundo diretrizes estabelecidas pela Sociedade Americana de Patologia Clínica Veterinária - ASVCP (Friedrichs et al., 2012), para que haja precisão aceitável nos valores de referência para dados laboratoriais, critérios de inclusão e exclusão de pacientes e amostras precisam ser cumpridos. Entre eles a quantidade de amostras. Stockham e Scott (2011) também acrescentam que devem ser utilizadas amostras coletadas de animais encaminhados para vacinação anual ou para procedimentos cirúrgicos eletivos. Neste trabalho, foram examinadas 187 amostras de gatos hígidos submetidos à cirurgia eletiva, tornando os valores encontrados confiáveis para utilização como intervalo de normalidade para a espécie felina.

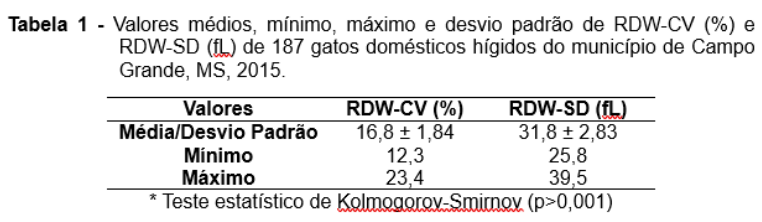

Os resultados obtidos de RDWCV neste estudo corroboram os valores encontrados por Jain (1993), Meyer e Harvey (2004), Riond et al.(2011), Silva (2013) e intervalos de referência publicados por duas universidades americanas (Universidade da Califórnia, Davis, 2011; Universidade de Medicina Veterinária de Cornell, 2014).

Entretanto, os intervalos de referência de RDW-SD, que independe do VCM e reflete melhor a variação no tamanho celular (Caporal e Comar, 2013), diferiram de Silva (2013) e Riond et al. (2011). Contudo, é importante considerar que esta divergência pode ser atribuída aos critérios metodológicos empregados. Silva (2013) utilizou 40 gatos domésticos provenientes de um gatil. Pequeno número de amostras e amostragem usando animais de gatis não são recomendadas visto que os felinos podem não possuir diversidade racial e/ou variações fisiológicas necessárias para intervalos de referência representativos (Stockham e Scott, 2011). Já Riond et al. (2011), tinham 0 objetivo de comparar 0 desempenho de dois equipamentos, e, portanto, não utilizou o estado sanitário 
como critérios de seleção dos animais para o estudo.

Os valores das médias e desvio padrão de RDW-CV e RDW-SD de acordo com o sexo estão apresentados na tabela 2 .

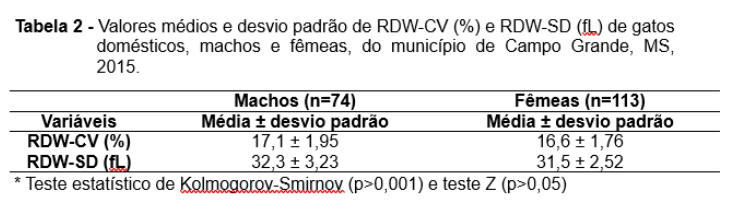

Não foi observada diferença estatística significativa quando os dados de RDW-CV e de RDW-SD (teste Z, $p>0,05)$ foram comparados entre machos e fêmeas, no presente trabalho, e comfirmam a constatação de que o sexo não interfere no diâmetro dos eritrócitos (Silva et al.,2015; Flaiban e Balarin, 2004; Souza et al., 2012; Balarin et al., 2001).

Os valores das médias e desvio padrão de RDW-CV e RDW-SD dos animais estudados de acordo com a idade estão apresentados na tabela 3. A análise estatística tanto dos valores de RDW-CV quanto do RDW-SD (teste Z, $p>0,05)$ comprovou ausência de diferença entre os grupos e, portanto, inexistência de influência da idade nestes parâmetros. Nenhum dos autores pesquisados apresentou dados estratificados por idade para felinos domésticos, contudo, estudos realizados em cães também constataram que a idade não interfere no diâmetro dos eritrócitos (Souza et al., 2012).

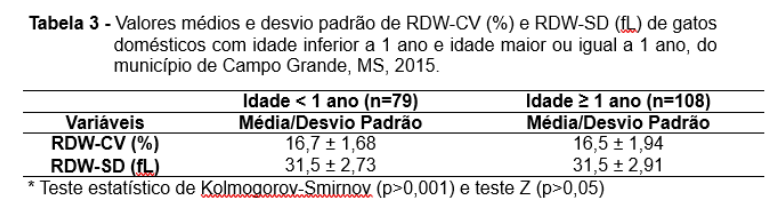

\section{CONCLUSÃO}

Os valores médios para RDW-CV e RDW-SD, apresentados no presente trabalho, pode ser usado como referência para felinos domésticos sem raça definida saudáveis, independente do sexo ou idade.

\section{REFERÊNCIAS}

BALARIN, M.R.S.; FONTEQUE, J.H.; SOUZA, C. et al. Valores da amplitude de distribuição do tamanho dos eritrócitos (RDW-Red cell distribution width) em equinos da raça puro sangue inglês (PSI) de ambos os sexos de 12 a 24 meses de idade. Semina: Ciências Agrárias, v.22, n.2, p.135-137, 2001.

CAPORAL, F.A.; COMAR, S.R. Evaluation of RDW-CV, RDW-SD and MATH-1SD for the detection of erythrocyte anisocytosis observed by optical microscopy. Jornal Brasileiro de Patologia e Medicina Laboratorial, v.49, n.5, p.324-331, 2013.

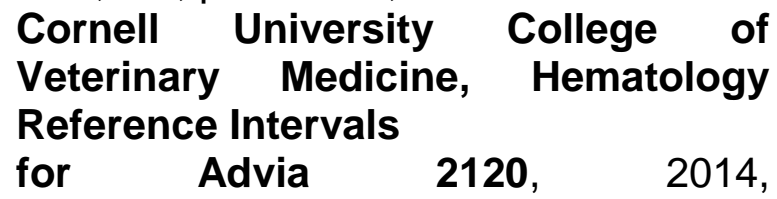
https://ahdc.vet.cornell.edu/sects/clinpat h/reference/hema.cfm.

FLAIBAN, K.M.C.; BALARIN, M.R.S. Estudo comparativo entre a amplitude de variação dos eritrócitos (RDW - Red Blood Cell Distribution Width) e o volume globular (VG), volume corpuscular médio (VCM) e a presença de anisocitose em extensão sangüínea em cães. Semina: Ciências Agrárias, v.25, n.2, p.125-130, 2004.

FRIEDRICHS, K.R.; HARR, K.E.; FREEMAN, K.P. et al. ASVCP Reference interval guidelines: determination of de novo reference intervals in veterinary species and other related topics. Veterinary Clinical Pathology, v.41, n.4, p.441-453, 2012. GONZÁLEZ, F. H. D.; CARVALHO, V.; MÖLLER, V. A. et al. Perfil bioquímico sanguíneo de cães e gatos na cidade de Porto Alegre, Rio Grande do Sul, Brasil. Arquivos da Faculdade de Veterinária, UFRGS, v. 29, n.1, p.1-6, 2001. 
HUNZIKER, S.; STEVENS, J., HOWELL, M.D. Red cell distribution width and mortality in newly hospitalized patients. The American Journal of Medicine., v.125, n.3, p.283-291, 2011. JAIN, N.C. Essentials of veterinary hematology. Philadelphia: Lea \& Febiger, 1993. 417p.

LAPPÉ, J.M.; HORNE, B.D.; SHAH, S.H. et al. Red cell distribution width, Creactive protein, the complete blood count, and mortality in patients with coronary disease and a normal comparison population. Clinica Chimica Acta, v.412, n. 20, p.2094-2099, 2011. MEYER, D.J.; HARVEY, J.W. Veterinary laboratory medicine: interpretation \& diagnosis. 2. ed. Philadelphia: Sauders, 2004. 351p.

RIOND, B.; WEISSENBACHER, R. H. L.; LUTZ, H. Performance evaluation of the Sysmex pocH-100iV Diff hematology analyzer for analysis of canine, feline, equine, and bovine blood. Veterinary Clinical Pathology, v. 40, p.484-495, 2011.

SALIBA, W.; BARNETT-GRINESS, O.; ELIAS, M.; et al. The association between red cell distribution width and stroke in patients with atrial fibrillation. The American Journal of Medicine., v.128, n.2, p.192, 2015.

SILVA, P. H. Amplitude de distribuição do diâmetro dos eritrócitos (RDW), volume corpuscular médio e reticulócitos em gato doméstico hígido (Felis catus Lennaeus, 1978). 2013. Presidente Prudente. Dissertação (Mestrado em Medicina Veterinária) - Curso de Pósgraduação em Medicina Veterinária Universidade do Oeste Paulista.

SILVA, P. H.; LAPOSY, C. B.; GIUFFRIDA, $R$. et al. Relationship between the red blood cell distribution width (RDW) and erythrocyte values in anemic and non-anemic cats. Semina: Ciências Agrárias, Londrina, v.36, n.5, p.3227-3232, 2015.
SLAUGHTER, E.; MOUNT, C. SCHOFIELD, C. Changes in Red Blood Cell Width Distribution as a Novel Biomarker for Bacteremia. Critical Care Medicine, v.41, 2013.

SOUZA, A.M.; CAMARGO, M.B.; BACELLAR, D.T.L. et al. Age and sex influence in canine red cell distribution width (RDW-CV and RDW-SD) values. Revista Brasileira de Ciência Veterinária, v.19, n.2, p.90-93, 2012.

STOCKHAM, S.L.; SCOTT, M.A. Fundamentos de Patologia Clínica Veterinária. 2.ed. Rio de Janeiro: Guanabara, 2011. 729p.

UC Davis Veterinary Medicine, Clinical Diagnostic Laboratory CBC Reference Intervals, 2011, http://www.vetmed.ucdavis.edu/vmth/loc al_resources/pdfs/lab_pdfs/UC_Davis_V $\mathrm{M} \overline{\mathrm{H}}$ _Hematology_Reference_Intervals.p df.

ZVORC, Z.; RAFAJ, R.B.; KULES, J. et al. Erythocyte and platelet indices in babesiosis of dogs. Veterinarski Arhiv, v.80, n.2, p.259-267, 2010. 